\title{
Applications of Optical Quantum Radiation Coherent States in Physical-Layer Security
}

\author{
Mark Herde and Said Mikki
}

\begin{abstract}
We explore applications of quantum antennas in wireless security by illustrating how the quantum-electromagnetic signature of optical radiation emitted by quantum antennas, the Schrodinger's coherent state structure, may be exploited to jointly design the transmitter and receiver of a $K$-ary digital communication link. We present a concrete example comprised of a quantum 64-QAM system including a complete description of the required general design principles developed from quantum mechanics and the quantum antenna's radiated coherent state structure. The simulation results illustrate improvement in spectral efficiency due to the use of $K=64$ over smaller values of $K$. We also compare the quantum antenna-based link performance with classical QAM utilizing classical antenna-based communication systems and report superiority of the quantum link over the classical version. To provide for security protection, our system is equipped with a quantum encryption protocol for quantum key distribution (QKD) and a demonstration of the complete quantum QAM system with encryption is presented. The main message of the paper is the fruitfulness of incorporating a multidisciplinary approach to our thinking about electromagnetic wireless systems through the joint deployment of electromagnetic, quantum, and communication theories in the design and development process of current and future advanced technologies.
\end{abstract}

\section{INTRODUCTION}

Quantum communication systems utilize quantum states as opposed to classical electromagnetic waves in order to carry information [1]-[4]. They are a possible answer to the neverending race to achieve faster and more reliable communication systems. While the bulk of research on quantum computing is currently still theoretical, quantum communication systems have proven time and again to be superior to their classical counterparts and several prototypes have been already constructed in the lab [5], [6]. Furthermore, quantum cryptography has offered nearly perfect security through the unique properties of quantum mechanics, e.g., the no-cloning theorem and entanglement [4]. In unison, a new era of communication systems is expected to arise, free of many issues we face with our current classical ones, especially lack of complete security [7], [8].

The way we think about security will need to change soon. Today's top encryption algorithms rely on functions which are not easily reversible. Due to the properties of these functions, it would be hard to try and find a key for decryption through brute force attack because it would take ordinary computers a long time to calculate [4]. Quantum computers pose a threat to many of these encryption methods, such as the Rivest-Shamir-Adleman (RSA) public-key cryptosystem [9]. This algorithm, like many others, is based on the practical difficulty of factoring the product of two large prime numbers. Shor's algorithm, specifically designed to run on a quantum

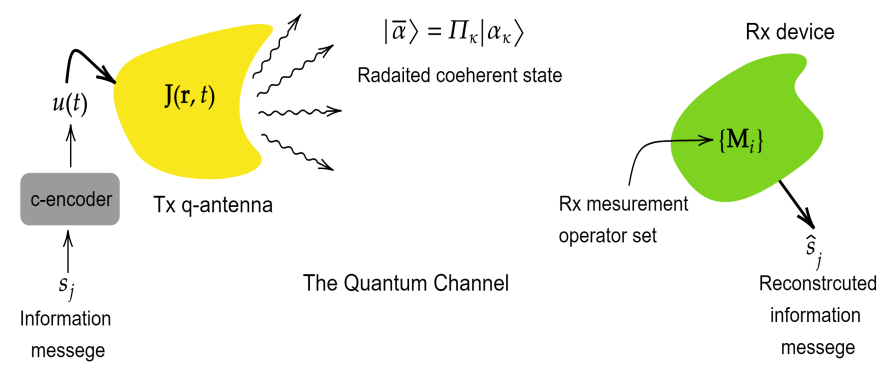

Fig. 1: The fundamental quantum coherent state antenna communication system. The classical encoder (c-encoder) and the Tx quantum antenna (q-antenna) together constitute the quantum encoder (q-encoder). On the receive side, a combination of the Rx q-antenna and signal processing is equivalent to the quantum mechanical measurement operator set $\left\{\mathbf{M}_{i}\right\}$. An essential component in the overall design process of the q-antenna communication system is to find the optimum or sub-optimum such operators matched to the coherent states radiated by the Tx q-antenna and resulting in minimum or near-minimum end-to-end probability of error.

computer, can increase the speed of factoring these products enough to make it feasible to break this encryption scheme in a matter of days [4], [10].

Due to the threat which quantum computers pose to modern cryptography, it is essential to explore new methods for keeping our information safe. Much research has gone into developing Quantum Key Distribution (QKD) protocols [4], [6], [11], based on using a symmetric key obtained from quantum communications. These methods provide information theoretic key agreement thanks to the laws of quantum mechanics. In particular, the BB84 algorithm relies on the Heisenberg Uncertainty Principle and has the added bonus of eavesdropper detection [4], [6].

There has been a growing interest in recent years in projecting into the classical description of electromagnetic systems, where one usually work from within the framework of the conventional system of Maxwell's equations [12]-[17], new elements derived from quantum physics. For example, some writers have looked into formulations integrating classical and quantum electromagnetics and explored differences between classical and nonclassical behaviour for application to fundamental theory, numerical methods, condensed-matter physics, material design, e.g., see [18]-[27].

One possible area where a injecting quantum ideas would be fruitful is the research subject of future antennas. This includes radiating devices with unusual behaviour which are 
expected to play a role in future applications. Examples are the recently introduced subject of nonlocal antennas [28]-[32], near-field antennas [33]-[48], nanoscale scattering/radiating systems [49]-[53], and plasma antennas [54].

In particular, within electromagnetic theory and its applications, quantum antennas present themselves as a promising emerging future antenna technology currently being investigated by several researchers, spanning a broad spectrum of applications including for example controlling quantum mechanical systems, quantum plasmonics, general control of quantum emitters, quantum nonreciprocity, coupling with quantum dots, quantum tomography and others [55]-[68]. In a majority of these papers, one may view research on quantum antennas as a natural evolution in which the earlier field of quantum emitters [49] is merged with the more recent subject of optical antennas [49], [69]. While the topic of quantum antennas is still quite broad and the main ideas related to their analysis and design are still taking shape, our focus here is on exploring one major use of these new radiators and how it can lend itself to fruitful practical applications in the field of secure wireless communications [67], [68].

A key physical aspect we believe is very useful for applying quantum antenna theory to wireless communications is the fact that quantum antennas excited by classical currents radiate what is called in quantum physics a coherent state of radiation [61]. In particular, it was recently proposed in [67] that modeling quantum optical radiation emitted by quantum antennas by coherent states is not only physically realistic, but also offers a unique advantage in which electromagnetics and communication theories can be simultaneously utilized to best design the system. This will be demonstrated in the present paper by actually constructing a practical quantum digital communication system with improved spectral efficiency by directly exploiting the structure of coherent states emitted by the quantum antenna. In a nutshell, the main objective of the present paper is introducing to a wide multidisciplinary audience the basic concepts and operating principles of a quantum antenna communication system based entirely on the use of coherent states of electromagnetic radiation emitted by classical source as proposed in [67].

The paper is organized as follows. In Sec. II, the main facts about coherent states needed to understand the construction of the quantum digital link are presented and the requisite theory pertinent to the examples to follow is briefly elaborated. ${ }^{1}$ The general quantum-mechanical principles of quantum communication systems are formulated in Secs. III and IV, where we adopt an approach based on quantum statistical decision theory. The general structures of the quantum modulators (transmitters) and demodulators (receivers) are explicated in detail with emphasis on how to set up the computational models that will be subsequently utilized in order to demonstrate a working system case study. The

\footnotetext{
${ }^{1} \mathrm{~A}$ complete theoretical analysis of the quantum-electrodynamic (QED) aspects of quantum antennas are outside the scope of the present paper but will be taken up by one of the authors in a separate paper. However, extensive references to literature on the physics of quantum radiation will be given throughout the paper in order to assist readers interested in learning more about the second quantization procedure at the heart of the production of quantum coherent states.
}

computational implementation of a concrete system is given in Sec. $\mathrm{V}$ where we focus on $K$-ary quantum digital link analysis with performance results reported for the increase of spectral efficiency concomitant with upping $K$ from 16 to 64 . Comparison with the classical QAM link is also provided and it is found that the quantum version is superior. As a further illustration of the practical use of the proposed system, we also demonstrate physical-layer security by directly applying a standard quantum encryption algorithm and quantum key distribution (QKD) to our construction. This is detailed in Sec. VI, where the process of converting binary information into radiated antenna quantum coherent states at the transmitter side is discussed, along with the information recovery process at the receive end while utilizing an efficient $K$-ary quantum QAM signaling scheme. Finally, we end with conclusion.

\section{Coherent Radiation by QuAntum Antennas}

\section{A. On the Electromagnetic Theory of Coherent States}

It has been shown as early as the beginnings of the $1960 \mathrm{~s}$ that a classical current sufficiently isolated from the backreaction of quantum radiation will produce pure coherent states [70], [71]. A coherent state, also sometimes known as Schrodinger's state (after their discover [72]) or Glauber's state (after their principal protagonist and popularizer [70]), can be represented mathematically by the ket $|\alpha\rangle$, an element of a suitable Hilbert space, where $\alpha \in \mathbb{C}$ is a complex number. This Hilbert space vector can be expanded in terms of the standard Fock's $n$-state bases of photon radiation $|n\rangle, n=1,2, \ldots$ (exactly $n$ light quanta excited) via the expansion [71]

$$
|\alpha\rangle=e^{-\frac{1}{2}|\alpha|^{2}} \sum_{n=0}^{\infty} \frac{\alpha^{n}}{\sqrt{n !}}|n\rangle .
$$

Further properties of coherent states have been developed very early in its history and can be found in several publications like [70], [71], [73]-[79]. By means of this expansion (1), one can effectively reduce computations with coherent states to calculations with Fock's state in order to facilitate applications to communication systems [67]. It is also possible to deploy coherent states themselves as basis to help expand every other state due to the fact that these special states are known to be complete. $^{2}$

Detailed discussion of the physical realization of the Tx quantum antenna is outside the scope of this paper which is more focused on expounding the general quantum-mechanical principles of the antenna link Rx terminal design, its overall end-to-end performance, and the application of coherent states to physical-layer security. However, the reader may consult the various different existing approaches proposed in the papers [55]-[60], [62]-[66] quoted in the Introduction above. We add here a promising candidate based on the use of optical antennas. Most researches on this type of radiators focus on receiving mode and integration with nearby nanoscale systems. Applications to wireless communications were suggested in [80]. One possible way to produce coherent states is to use a subwavelength laser beam [81] directly focused at the gap

\footnotetext{
${ }^{2}$ In fact, coherent states are overcomplete, see [71] for a rigorous treatment.
} 


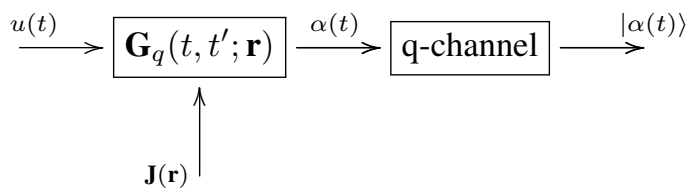

Fig. 2: The fundamental structure of the q-antenna problem proposed in [67]. The functions $\mathbf{J}(\mathbf{r}), u(t)$ and $\alpha(t)$ are classical. The input real signal $u(t)$ encodes information to be transmitted via the quantum channel (q-channel). On the other hand, the complex signal $\alpha(t)$ is rooted in the electromagnetic process of quantum radiation whereupon a coherent quantum state $|\alpha(t)\rangle$, an element of the Fock (Hilbert) space of the problem, is produced by a classical current source $u(t) \mathbf{J}(\mathbf{r})$. The qGreen's function $\mathbf{G}_{q}\left(t, t^{\prime} ; \mathbf{r}\right)$ represents the linear-system-like properties of the antenna and can be derived from specific models of the field-matter Hamiltonian of the q-antenna.

of an optical nano-wire antenna [82]. The antenna excited in this way was shown to exhibit a stable radiation pattern and its efficiency and gain characteristics were found to improve with optimization of the antenna shape [83]. Since intense subwavelength beams are locked up with the resonant plasmonic surface waves excited by the laser field, this structure may provide a practical realization of an externally-controlled classic current shielded from photon back-reaction [83], leading then to the radiation of coherent states. Subsequently, the well-known ability of laser sources to be directly or indirectly modulated by external driving pulse sources [84] allows easy insertion of an information-carrying time-domain excitation signal $u(t)$ by modulating the subwavelength beam directed at the dipole antenna's gap.

In Fig. 1 we show the main configuration of the quantum antenna system implementation of a quantum wireless link. The goal is to send classical information over a quantum channel. The antenna is excited by a classical time-waveform $u(t)$, which is generated by source coding the information massage $s_{j}$ via the classical encoder (c-encoder). The timedomain signal in turn generates a classical radiating current distribution $\mathbf{J}(\mathbf{r}, t)$. As an optical antenna, this current will radiate a quantum (coherent) state (often a multimode coherent state $|\bar{\alpha}\rangle$, see (7) and (9) below), which then is used to convey information into the receive quantum antenna. In this paper, the receive device is modeled as a quantum measurement operator set $\left\{\mathbf{M}_{i}\right\}$. Physically, a receive antenna should interact with quantum radiation (the generated coherent state) in order to produce a meaningful signal at the final receive port, the signal $\hat{s}_{j}$ in Fig. 1. From the wireless communication viewpoint, however, a meaningful signal is interpreted as the (best possible) reconstruction of the information signal (message) originally injected into the input port of the Tx q-antenna via the signal $u(t)$. Therefore, in this paper we view the problem of designing the Rx q-antenna as finding the optimum or near-optimum set of measurement operators $\left\{\mathbf{M}_{i}\right\}$ that would minimize the probability of error of end-toend transmission per symbol.

We will now briefly explain how coherent states originate from the quantization of field-matter interactions. The Hamiltonian of the Tx quantum antenna can be written as

$$
H=H_{\text {em,free }}+H_{\text {int }},
$$

where the in radiation gauge the free-field electromagnetic Hamiltonian can be expanded into the following timeindependent normally-ordered form

$$
H_{\mathrm{em}, \text { free }}=\int_{\mathbf{k} \in \mathbb{R}^{3}} \frac{\mathrm{d}^{3} k}{(2 \pi)^{3}} \hbar \omega_{k} \sum_{s \in\{0,1\}} a_{\mathbf{k} s}^{\dagger} a_{\mathbf{k} s} .
$$

The reduced Planck constant is $\hbar:=h / 2 \pi$, where $h$ is Planck constant. The superscript $\dagger$ indicates the operation of taking the adjoint (Hermitian operation or complex transpose for matrices). The free-space dispersion relation is $\omega_{k}=$ $c k, k:=|\mathbf{k}|$. The non-Hermitian operators $a_{\mathbf{k} s}^{\dagger}$ and $a_{\mathbf{k} s}$ are the creation and annihilation operators, respectively, of the mode with wavevector $\mathbf{k} \in \mathbb{R}^{3}$ and one of the two orthogonal polarizations transversal to $\mathbf{k}$ indexed by $s \in\{0,1\}$. We have also removed the global ground state constant level term $\hbar \omega_{k} / 2$ from the free Hamiltonian (3) since it does not contribute to transition energies induced by the external current source [78].

The interaction Hamiltonian $H_{\text {int }}$ in quantum electrodynamics is written in terms of the gauge field $\mathbf{A}(\mathbf{r}, t)$, the magnetic vector potential field (we assume no external charge density for simplicity.) Its standard expression is [79]

$$
H_{\text {int }}(t)=-\int \mathrm{d}^{3} r \mathbf{J}(\mathbf{r}, t) \cdot \mathbf{A}(\mathbf{r}, t) .
$$

Within the hemiclassical approximation, the antenna current source current distribution $\mathbf{J}(\mathbf{r}, t)$ is treated as a classic function (not operator), while the radiated field is promoted to operator field form. The Hermitian quantum field operator A is expressed as

$\mathbf{A}(\mathbf{r}, \mathbf{t})=\mathbf{A}^{(+)}(\mathbf{r}, t)+\mathbf{A}^{(-)}(\mathbf{r}, t)=\mathbf{A}^{(+)}(\mathbf{r}, t)+\left[\mathbf{A}^{(+)}(\mathbf{r}, t)\right]^{\dagger}$.

In the radiation gauge, the positive-frequency component can be expanded into creation and annihilation operators sum in the form

$$
\mathbf{A}^{(+)}(\mathbf{r}, t)=\int_{\mathbf{k} \in \mathbb{R}^{3}} \frac{\mathrm{d}^{3} k}{(2 \pi)^{3}} \sqrt{\frac{\hbar}{2 \varepsilon_{0} \omega_{k}}} \sum_{s \in\{0,1\}} \hat{e}_{\mathbf{k} s} a_{\mathbf{k} s} e^{\mathrm{i}(\mathbf{k} \cdot \mathbf{r}-\omega t)},
$$

where $\hat{e}_{\mathbf{k} s}, s=0,1$ are two orthogonal unit vectors (possibly complex) satisfying $\hat{e}_{\mathbf{k} s} \cdot \mathbf{k}=0$ for all $\mathbf{k}$ and $s$ and describe the polarisation of the $\mathbf{k} s$ photon. The quantity $\varepsilon_{0}$ is the free-space electric permitivity.

After the use of perturbation theory to solve the coupled field-matter problem within the hemiclassical approximation, it can be shown that the radiated state acquires the remarkably succinct form of a multimode coherent radiation state $|\bar{\alpha}\rangle$ given by

$$
|\bar{\alpha}\rangle=\prod_{\mathbf{k} s}\left|\alpha_{\mathbf{k} s}\right\rangle
$$


where the product state is formed over all wavevectors $\mathbf{k}$ and polarizations $s=1,2$. Here, we have [71], [79]

$$
\left|\alpha_{\mathbf{k} s}\right\rangle=e^{-\frac{1}{2}\left|\alpha_{\mathbf{k} s}\right|^{2}} \sum_{n_{\mathbf{k} s}=0}^{\infty} \frac{\alpha_{\mathbf{k} s}^{n}}{\sqrt{n_{\mathbf{k} s} !}}\left|n_{\mathbf{k} s}\right\rangle,
$$

where $\left|n_{\mathbf{k s}}\right\rangle$ denotes the Fock state representation (number of light quanta) in the ksth mode. ${ }^{3}$ Special cases of (7) were discussed in some references, e.g., see [70], [71], [78], [85], [86]. The detailed full analysis will be given in a separate publication since it is lengthy and the content is not needed for the topic of this paper. We know however that by carrying out the full details of the perturbation solution, it can be shown that a Green's function $\mathbf{G}_{q}$ connecting the current source $\mathbf{J}(\mathbf{r}, t)$ and the radiated coherent state must exist [67]. The structure of this Green's function is summarized in Fig. 2. The quantity $\mathbf{G}_{q}$ is not needed for constructing and analyzing the complete quantum antenna communication system since in this paper we design the system (modulator and demodulator) by operating directly with (7).

\section{B. On the Communication Theory of Coherent States}

In this paper, we deploy Helstrom' theory of quantum communications, which applies to both digital and analog quantum communication schemes [1] and will be reviewed and reformulated for our purposes in Sec. IV. In order to explore the ability of our proposed quantum coherent-state system to increase spectral efficiency, we focus on investigating the performance of a digital multi-symbol transmission scheme, the $K$-ary system [87]-[89]. In general, for an $K$-ary digital communication link, one is interested in sending $K$ symbols encoded by $K$ different states $\rho_{1}, \rho_{2}, \ldots, \rho_{K}$ [87]. Here, each $\rho_{i}$ is the standard quantum density (Hilbert space) operator associated with the corresponding quantum (Hilbert space) state indexed by $i$ [90]-[92]. This process is here termed ' $\mathrm{q}$ encoding' and is captured by Fig. 3 [68].

Digital information may be formed via a quantum encoding scheme in which each complex number $\alpha_{\mathbf{k} s}$ is assigned one sub-symbol (vector modulation [93]). That is, within the framework of a finite-dimensional approximation of (7) in $K$ dimensions, we may write in general [68]

$$
|\bar{\alpha}\rangle=\left|\alpha_{1}\right\rangle \otimes\left|\alpha_{2}\right\rangle \otimes \cdots \otimes\left|\alpha_{K}\right\rangle,
$$

where $\alpha_{m} \in \mathbb{C}, m=1,2, \ldots, K$, is the complex amplitude of the coherent state representation of the $m$ th mode. ${ }^{4}$ For scalar modulation, each one of the $K$ symbols is directly assigned a distinct $|\alpha\rangle$ where the entire radiated state is written as a single coherent state (one-mode theory). In this paper, we focus for simplicity on a single-mode coherent state representation and hence use scalar modulation for implementing the $K$-ary digital communication system application.

\footnotetext{
${ }^{3}$ The total q-antenna Fock space is the tensor product of all such modal Fock spaces.

${ }^{4}$ On the other hand, q-antenna theory may provide an alternative, more sophisticated method to form the q-encoding mapping of Fig. 3 through current source engineering [67], a subject outside the scope of the present work.
}

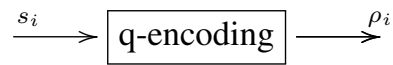

Fig. 3: The process of mapping $M$ classical symbols to $M$ q-states in q-communications using a specially designed qantenna. Here, the radiated states are represented in terms of density operators $\rho_{i}$.

\section{Construction of the QuAntum Communication SYSTEM}

\section{A. General Overview of the Communication Link}

We begin with the construction of the quantum communication system to be used and break it down into parts to understand it. The system to be analyzed is shown in Fig. 4. The system begins with the information source named Alice

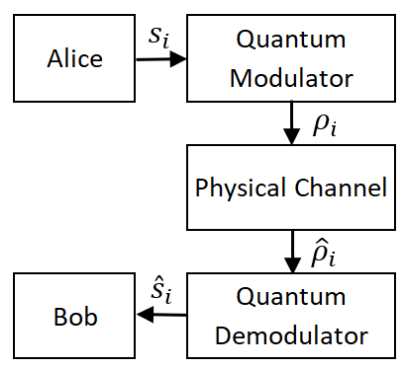

Fig. 4: General construction of the communication system without encryption. The system is much like classical communications but with components which utilize quantum states.

by convention. Alice sends a message $s_{i}$ to be sent to a user on the other end of the channel. The message is changed into a form suitable for travelling through the physical channel by the modulator. This q-modulated state $\rho_{i}$ then radiates through the channel acting as an information carrier. Just as in classical communications, the quantum signal loses its integrity after being transmitted through the channel. On the other end of the channel is a demodulator which attempts to reconstruct the tampered signal. This receiver is illuminated by a quantum state whose density operator is denoted by $\hat{\rho}_{i}$. We design the quantum demodulator based on the modulator choice such that the receiver of the q-link will output the symbol most likely to have been sent based on the noisy signal, i.e., the quantum estimation $\hat{s}_{i}$ [1]. The converted quantum state is then sent to the end user, Bob, to be reconstructed into its original format.

\section{B. The Quantum Modulator (the Encoder-Antenna System)}

We will consider a $K$-ary digital communication system, where the information source emits symbols from a predefined set which we call the alphabet $\mathcal{A}$. We can enumerate the output of the information source as $s_{i} \in \mathcal{A}, i=1 \ldots K$. To begin preparing the symbols for transmission, the first part of the modulator will be a quantum encoder (q-encoder). This device realizes a mapping of the symbols in $\mathcal{A}$ to pure quantum states, $\left|s_{m}\right\rangle$. The states can also be represented by the density operator notation

$$
\rho_{i}=\left|s_{i}\right\rangle\left\langle s_{i}\right| .
$$


There is thus a second alphabet composed of quantum states which the q-encoder maps the symbols to. We call this alphabet the state constellation, $\mathcal{A}_{q}$. We can then express the functionality of the q-encoder as

$$
s_{i} \in \mathcal{A} \stackrel{\text { q-encoding }}{\longrightarrow} \rho_{i} \in \mathcal{A}_{q}
$$

The cardinalities of $\mathcal{A}$ and $\mathcal{A}_{q}$ in general do not need to be equal. However, the case of $|\mathcal{A}|<\left|\mathcal{A}_{q}\right|$ would not make sense because there would inevitably be unused symbols in the constellation. Therefore, the q-encoder carries out a surjective mapping, meaning the relationship $|\mathcal{A}| \geq\left|\mathcal{A}_{q}\right|$ holds. In this paper, we are going to assume that the cardinalities are the same, so that our q-encoder carries out the bijective mapping $s_{i} \rightarrow \rho_{i}$.

While the q-encoder maps a symbol to a quantum state, it cannot send the state through the channel. Instead this can be done with a quantum antenna (q-antenna), which radiates a pure quantum state based on a suitable excitation as explained in Sec. II. The q-encoder will drive the q-antenna by outputting a pulse, $s_{i}(t)$, which will cause the q-antenna to radiate the pure state $\rho_{i}$.

The final construction of the quantum modulator is shown in Fig. 5. The q-encoder and q-antenna are thus designed hand-

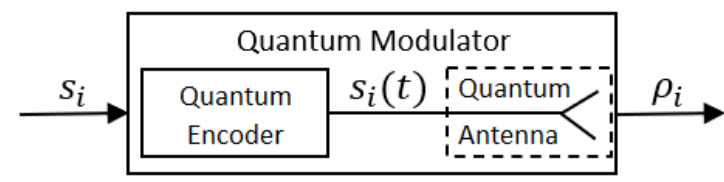

Fig. 5: Construction of the modulator for a quantum communication system.

in-hand; however, the construction of the q-antenna and what pulses drive it are outside the scope of this paper. In this paper, we will explore the bijective mapping in a high-level view, assuming this device can output any state we wish.

\section{The Quantum Demodulator}

On the opposite side of the channel we have the demodulator which takes as an input the transmitted state after going through the channel, $\hat{\rho}_{i} \in \mathcal{A}_{q}$, and outputs the symbol which it believes was sent, $\hat{s}_{i} \in \mathcal{A}$. There are two primary components necessary for this to work: quantum measurement and decision devices. The quantum measurement device realizes a projective mapping of the incoming state onto the global Hilbert space of the overall q-antenna communication system, which will be explained in more detail in Sec. IV. Since we design the modulator to output whatever quantum states we want, the construction of the measurement device is tied directly with the design of the modulator.

We can model the measurement device as an observable, $M$. By the spectral decomposition theorem [4], [90], [94], we can decompose this observable as

$$
M=\sum_{i=1}^{K} \lambda_{i} P_{i},
$$

where the members of the set $\left\{P_{i}\right\}_{i=1}^{K}$ form a projector system. The design of the measurement device thus entails optimizing this projector system to measure symbols from $\mathcal{A}_{q}$. That is, the output of $M$ will be fed to the decision device, denoted by $D(\lambda)$. This will take the output of $M$ and make a decision of which symbol was transmitted. Since we chose $|\mathcal{A}|=\left|\mathcal{A}_{q}\right|$, the function of this device can be compactly written as

$$
D\left(\lambda_{i}\right)=\hat{s}_{i} .
$$

With this description the quantum demodulator can be represented as shown in Fig. 6.

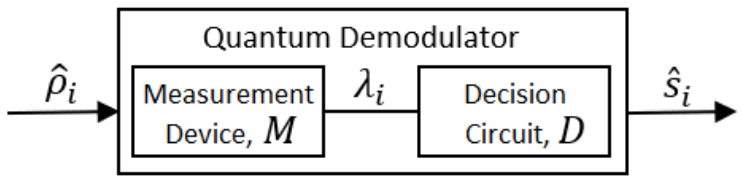

Fig. 6: Construction of the demodulator for a quantum communication system.

The design process resulting in complete determination of the decision device $D(\lambda)$ will be elaborated in Sec. IV where we recruit Helstrom statistical decision theory in order to find optimum or sub-optimum Rx q-antenna-decoder representations in terms of the measurement operators $M_{i}$.

\section{QuAntum Communication System DESIGN}

The design of this system requires a good background of quantum concepts applied to communications. ${ }^{5}$ Here, we adopt the approach detailed in [1], [96]-[98], which utilizes quantum statistical decision theory in order to design the receiver. Starting from the beginning of our system, we have the construction of the quantum modulator which is all about modulating coherent states for sending them through the channel. We then move on to the demodulator which will take our modulated coherent states and measure them to figure out what information was being sent. The design of the demodulator is directly tied to how we decide to modulate the coherent states we send. In this Section, we investigate a quantum version of Quadrature-Amplitude Modulation (QAM) using coherent states.

\section{A. Quadrature Amplitude Modulation (QAM) Using Radiated Optical Coherent States}

In the $K$-ary communication system we are modelling, Alice will encode each symbol from her alphabet $\mathcal{A}$ into a pure coherent state to send through the channel. A coherent state $|\alpha\rangle$ is defined by (1) where the enumerable set $|n\rangle$ are the radiation Fock number states (computational basis). The complex parameter $\alpha$ has the meaning of photonic intensity; in particular, the value $|\alpha|^{2}$ is the average number of photons

\footnotetext{
${ }^{5}$ Good general background in quantum theory can be found in [85], [91], [92]. For expositions of quantum theory geared toward quantum computing and information, see [4], [10]. Specialized treatment of communication systems through quantum physics may be found in places like [2], [3]. Readers interested in a rigorous exposition of the mathematical foundations behind quantum theory may consult [94], [95].
} 
in the state $|\alpha\rangle$ [70], [71], [79]. The collection of quantum symbols used for encoding is the state constellation, which we write as

$$
\mathcal{A}_{q}=\left\{\left|\alpha_{i}\right\rangle\right\}, i=1, \ldots, K .
$$

With this definition we see that the modulator will essentially take a set of bits and map them to a complex number $\alpha_{i}$ which is converted into a coherent state $\left|\alpha_{i}\right\rangle$. We will call the set of complex parameters associated with the state constellation $\mathcal{A}_{q}^{*}=\left\{\alpha_{i}\right\}$.

In our application of this communication system, we will transmit symbols using Quantum QAM with coherent states. The QAM alphabet is simply a square grid of $K=L^{2}$ equally spaced complex values. We first introduce the $L$-ary balanced alphabet [87]

$$
\mathcal{A}_{\mathcal{L}}=\{-(L-1)+2(i-1) \mid i=1,2, \ldots, L\} .
$$

The set of complex numbers which create the $K$-ary QAM grid is then defined by

$$
\mathcal{A}_{\mathrm{QAM}}=\left\{\Delta(u+i v) \mid u, v \in \mathcal{A}_{\mathcal{L}}\right\},
$$

where the parameter $\Delta \in \mathbb{R}$ is a scaling factor which relates to the transmitted operating power of the communication system. For this system we simply set $\mathcal{A}_{q}^{*}=\mathcal{A}_{\mathrm{QAM}}$ which formally defines the set of complex parameters used by our q-encoder to realize our state constellation $\mathcal{A}_{q}$. Recalling that we have a binary stream of information to send to Bob, we can take sets of $\log _{2}(K)$ bits from this stream and treat them as binary numbers. These binary numbers can then be used to index the set of complex parameters which ultimately maps each set of bits to a coherent state from the state constellation.

\section{B. The Process of Quantum Measurement at the Receive End}

We now move on to the primary mechanism behind our receiver: quantum measurements of radiated optical coherent states. The most basic type of quantum measurement was introduced by Von Neumann, and they are called projective measurements [90]. They work inside a Hilbert space $\mathcal{H}$, which is basically the proper linear vector space arena inside which quantum states live [94], [95]. The overall procedure adopted in this paper will be summarized through the following steps.

1) The Projector System: We start by selecting a sufficient set of operators called projectors enjoying a special set of properties. In particular, a projector $\mathbf{P}$ is Hermitian and idempotent if it satisfies the following two properties, respectively [1], [2], [90]:

$$
\mathbf{P}=\mathbf{P}^{\dagger}, \quad \mathbf{P}=\mathbf{P}^{n},
$$

where $n$ is an integer. Further, it can be shown that the eigenvalues of projectors are either 0 or 1 , meaning projectors are positive semidefinite, i.e., they satisy

$$
\langle\alpha|\mathcal{P}| \alpha\rangle \geq 0, \forall|\alpha\rangle \in \mathcal{H} .
$$

These properties give the projectors unique capabilities which lend themselves perfectly to be used as measurement devices.
Projective measurements are carried out using a projector system

$$
\left\{\mathbf{P}_{i}, i=1,2, \ldots, M\right\} .
$$

A projector system is a set of projectors such that they resolve to the identity of their Hilbert space and they are orthogonal:

$$
\mathbf{P}_{i} \mathbf{P}_{j}=\delta_{i j} \mathbb{1}_{\mathcal{H}}, \quad \sum_{i} \mathbf{P}_{i}=\mathbb{1}_{\mathcal{H}},
$$

where $\delta_{i j}$ is the Kronecker delta function and $\mathbb{1}_{\mathcal{H}}$ is the identity operator of the Hilbert space $\mathcal{H}$. The projector fully defines the statistics of a measurement system with a total of $M$ possible outcomes. The probabilities of the $i$ th outcome in its eigenstate state $\left|\alpha_{i}\right\rangle$ is given by

$$
P(i)=\left\langle\alpha_{i}\left|\mathbf{P}_{i}\right| \alpha_{i}\right\rangle,
$$

where $\left|\alpha_{i}\right\rangle$ is the state to be measured.

2) Positive Operator Valued Measures: Quantum measurements can be generalized from the basic projector system defined above into using Positive Operator-Valued Measurements (POVMs) [4]. While still a set of Hermitian operators, they are not necessarily projectors. A system of POVMs is a set of operators, $\left\{\mathbf{Q}_{i}\right\}$, which are required to have the following properties [1], [4], [6], [11]

(i) Hermitian: $\mathbf{Q}_{i}^{\dagger}=\mathbf{Q}_{i}$,

(ii) Positive semi-definite: $\mathbf{Q}_{i} \geq 0$,

(iii) Resolve identity: $\sum_{i} \mathbf{Q}_{i}=I$.

This retains the properties necessary for performing quantum measurements while allowing for more flexibility in the design process [1], [4], [99]. In particular, with POVMs we are no longer concerned with the particular outcome of the measurement, but instead we can simply work with their probabilities. In fact, we retain the same type of format for probability of measurement given by

$$
P(i)=\left\langle\alpha_{i}\left|\mathbf{Q}_{i}\right| \alpha_{i}\right\rangle,
$$

which should be compared with (20).

An example of obtaining a system of POVMs would be to start with a projector system for a particular measurement device, $\bar{M}=\left\{\mathbf{P}_{i}\right\}$. To form the POVM system, one may partition the alphabet of measurement outcomes obtained with the projector system and form a set of operators from the partitions. Partitioning the system into $\bar{M}_{1}, \bar{M}_{2}, \ldots, \bar{M}_{L}$, a system of $L$ POVMs are formed and given by

$$
\mathbf{Q}_{i}=\sum_{j \in \bar{M}_{i}} \mathbf{P}_{j} .
$$

This clearly illustrates how moving from projectors to POVMs constitutes an increase in abstraction that nevertheless improves the ability to actually realize the final quantum link receiver design since several projectors may be grouped into a single POVM that might be easier to implement.

3) Square Root Measurements (SRM): We now briefly talk about a measurement technique which allows us to figure out an optimized valid set of POVMs for a particular set of quantum states. Square root measurement (SRM) [100] is based on finding a set of measurement vectors $\left|\mu_{i}\right\rangle$ which we can use to construct an apparatus for measuring some set of 
states, $\left|\gamma_{i}\right\rangle$, e.g., see some of its applications in [2], [99], [101], [102]. Defining the error between a state and its corresponding measurement vector as

$$
\left|e_{i}\right\rangle=\left|\alpha_{i}\right\rangle-\left|\mu_{i}\right\rangle,
$$

SRM seeks $\left|\mu_{i}\right\rangle, i=1, . ., K$, which minimize the quadratic error

$$
\mathcal{E}=\sum_{i=1}^{K}\left\langle e_{i} \mid e_{i}\right\rangle
$$

subject to the constraint of the resolution of the identity

$$
\sum_{i=1}^{K}\left|\mu_{i}\right\rangle\left\langle\mu_{i}\right|=\mathbb{1}_{\mathcal{H}} .
$$

To perform calculations, we introduce the state matrix $\Gamma$ defined as the collection of kets from the state constellation:

$$
\Gamma=\left[\left|\alpha_{1}\right\rangle\left|\alpha_{2}\right\rangle \ldots\left|\alpha_{K}\right\rangle\right],
$$

where each ket forms a column in $\Gamma$. We can then define the Gram matrix

$$
G:=\Gamma^{*} \Gamma
$$

which is the $K \times K$ matrix of inner products of the constellation of states with general element

$$
G_{i j}=\left\langle\alpha_{i} \mid \alpha_{j}\right\rangle, i, j=1,2, \ldots, K .
$$

It can be shown that Gram's Matrix is actually a matrix of transition probabilities [2]

$$
p_{c}(j \mid i)=\left|\left(G^{\frac{1}{2}}\right)_{i j}\right|^{2} .
$$

With this, we can directly arrive at the minimum probability of error yielded from SRM calculation:

$$
P_{e}=1-\frac{1}{K} \sum_{i=1}^{K}\left|\left(G^{\frac{1}{2}}\right)_{i i}\right|^{2} .
$$

Therefore, by taking the eigenvalue decomposition of the Gram matrix,

$$
G=V D V^{*},
$$

one may simply calculate

$$
G^{\frac{1}{2}}=V D^{\frac{1}{2}} V^{*}
$$

in order to eventually estimate (30). The POVMs come from the columns of the matrix $V$, while the matrix $D$ gives the corresponding outcomes related to measuring a particular state with a POVM from the produced set.

\section{COMPUTATIONAL IMPLEMENTATION OF THE QUANTUM QAM COHERENT STATE SYSTEM}

Now that we have a firm grounding in the mechanics of how this system is designed, we will discuss the metric we use to determine how well our system is operating. At any point during communication, Alice may send a message which is converted into complex parameter $C \in \mathcal{A}_{q}^{*}$. The apriori probability of this parameter being chosen is $p_{i}:=P\{C=$ $\left.\alpha_{i}\right\}$. Further, the average number of photons per symbol in the resulting quantum state will be $\left|\alpha_{i}\right|^{2}$. With this we introduce

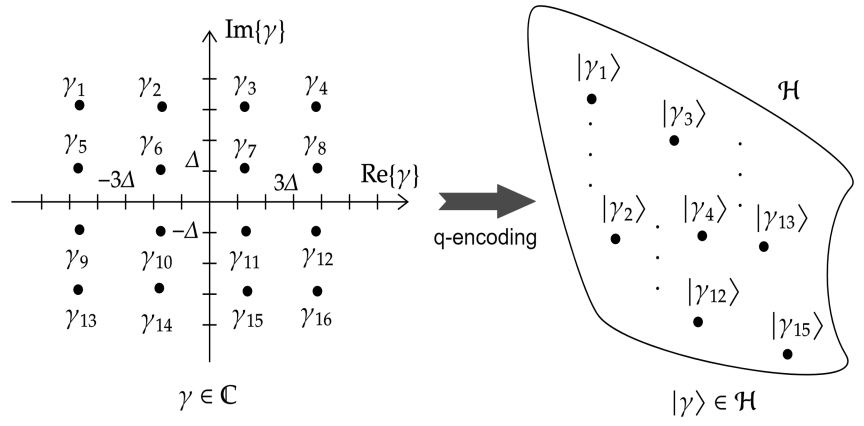

Fig. 7: (Left) 16-QAM constellation space $A_{q}^{*}$ in the complex plane $\mathbb{C}$. Each of the 16 symbols is encoded by the complex number $\gamma_{i}, i=1,2, \ldots, 16$ (not all symbols labels are showing in the right figure). The scale factor $\Delta$ can be used to obtain the physical values of each symbol $\gamma_{i}$ according to the abstract geometric configuration in the figure. (Right) The actual Hilbert space constellation $A_{q}$ of the q-channel (Fock space of the quantum antenna system.)

the signal photons per symbol $N_{S}$ defined by the expected value

$$
N_{S}=\mathbb{E}\left\{|C|^{2}\right\}=\sum_{i} p_{i}\left|\alpha_{i}\right|^{2},
$$

which is equivalent to the average number of photons necessary for using this state constellation for communication. Assuming equiprobable symbols, this simply becomes

$$
N_{S}=\frac{1}{K} \sum_{i}\left|\alpha_{i}\right|^{2} .
$$

The paramter $N_{S}$ will help us understand how well the communication system perform relative to other functionally similar systems, e.g., the classical QAM-based link.

We may now take this one step further to make it easier computationally to investigate the performance with multiple symbols. Let us express the complex symbols in the form

$$
\overline{\mathcal{A}}_{q}^{*}=\left\{\bar{\alpha}_{i}\right\},
$$

such that the parameters $\bar{\alpha}_{i}$ are normalized complex symbols, where $\alpha_{i}=\Delta \bar{\alpha}_{i}$. This allows us to rewrite our state constellation in terms of the factor $\Delta$ in (15) as follows:

$$
\mathcal{A}_{q}^{*}=\left\{\Delta \bar{\alpha}_{i}\right\} .
$$

Now introducing the form factor of a state constellation

$$
\mu_{K}:=\frac{1}{K} \sum_{i}\left|\bar{\alpha}_{i}\right|^{2},
$$

we can rewrite the signal photons per symbol by swapping the original complex parameters for the normalized symbols and substituting the form factor

$$
N_{S}=\Delta^{2} \mu_{K} .
$$

It is also worth adding that since in $K$-ary modulation scheme there are $\log _{2} K$ bits per each symbol, the signal photon count per bits $N_{R}$ is simply given by $N_{R}=N_{S} / \log _{2} K$. 


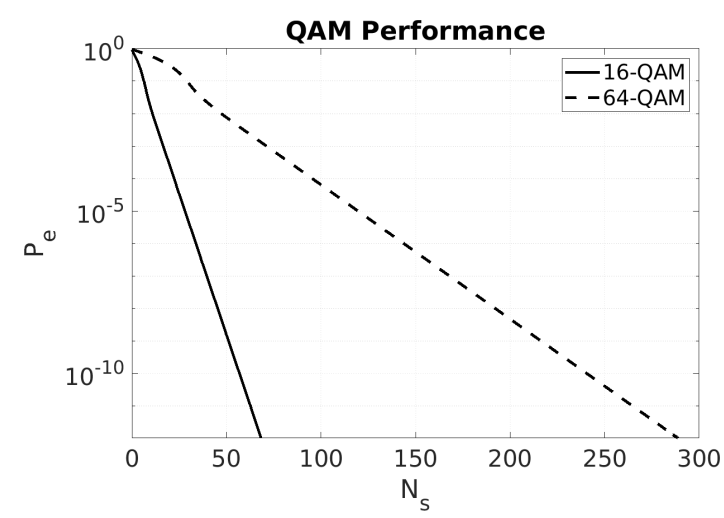

Fig. 8: Error probability $P_{e}$ vs. signal photons per symbol $N_{S}$ for 16- and 64-QAM.

Fig. 7 illustrates an example of a quantum QAM system signal constellation. In the left, we show the 16 "signals" representing the QAM symbol alphabet $\mathcal{A}$, i.e., the appropriate constellation of complex numbers belonging to $A_{q}^{*}$. These are the complex numbers $\gamma_{i}, i=1, \ldots, 16$, that will be encoded by the quantum antenna producing the radiation (coherent states ) $\left|\gamma_{i}\right\rangle, i=1, \ldots, 16$, as per Sec. II. The generalization to arbitrary number of symbols is straightforward and follows the same construction shown in Fig. 7 (left) by means of the formula (15). In our case, the quantum encoding becomes nothing but quantum antenna radiation. These quantum states live in the Hilbert space of the quantum antenna system (Fock space) and are shown in Fig. 7 (right). Note that the positions of vectors $\left|\gamma_{i}\right\rangle$ in $\mathcal{H}$ need have no geometrical symmetry like those enjoyed by $\gamma_{i} \in \mathbb{C}$ in Fig. 7 (left). The Hilbert space $\mathcal{H}$ is theoretically infinite dimensional. Computationally speaking, if there is no thermal noise we need only to operate with the complex numbers $\gamma_{i}$. Indeed, in the absence of thermal noise the radiation states are pure coherent states. Consequently, we may then use the exact inner product formula [71], [78], [79]

$$
\left\langle\gamma_{i} \mid \gamma_{j}\right\rangle=\exp \left\{-\frac{1}{2}\left(\left|\gamma_{i}\right|^{2}+\left|\gamma_{j}\right|^{2}-2 \gamma_{i}^{*} \gamma_{j}\right)\right\}
$$

to compute the Gram matrix (27) via (28).

Putting everything together, the entire transmitter-receiver link was simulated using a channel that contains only shot noise (no thermal noise). Note that shot noise represents the intrinsic quantum noise of any quantum communication system. Using Quantum $K$-ary QAM with coherent states and utilizing the SRM technique described above in order to obtain optimal POVMs for the resulting state constellation, it is possible to produce an error probability measure estimation using the Gram matrix formula (30). This measure can be directly compared with simulation experiment. Indeed, After completing the design process and obtaining the Rx measurement operators $M_{i}$, we can set up a complete simulation of the quantum link and hence confirm the probability of error estimation. The idea is to simply count and average the number of errors made at the receiver by comparing the reconstructed signal (after performing quantum measurement) with the original coded signal generated at the transmitter side. Furthermore, we can vary the number of photons per symbol using the scaling factor $\Delta$ introduced in our alphabet of complex parameters $\mathcal{A}_{q}^{*}$. This allows us to directly find how the symbol error rate is impacted by increasing the "signal-tonoise-ratio" (SNR), which in our case is directly proportional to the parameter $N_{S}$.

Complete simulation results for two benchmark cases of 16and 64-QAM quantum links are shown in Fig. 8 where the symbol probability of error $P_{e}$ is computed against variable signal photons per symbol $N_{S}$. Note that our measure of performance is based on the power usage of the different QAM constellation, meaning that the symbol rate is fixed. The 64QAM system send more information per symbol period and so the spectral efficiency is enhanced whenever $K$ is successfully increased [87]. While more information is sent per symbol for higher $K$, on average a higher $K$ also means more power is consumed for equiprobable symbols. This nicely illustrate the well known trade-off between spectral efficiency and power for the same level of probability of error that is also observed in classical links [88], [103], [104].

What about the relative performance of quantum link to the classical channel? What we can also show is that the quantum QAM consistently outperforms its classical version. In order to conduct this comparison, we first need to build the classical QAM system. It can be easily shown that in terms of the signal photons per symbol, the classical QAM error performance is given by

$$
P_{e, \text { classical }}=1-\left[1-2\left(1-\frac{1}{\sqrt{K}}\right) Q\left(2 \sqrt{\frac{N_{S}}{\mu_{K}}}\right)\right],
$$

where $Q$ is the standard error function. To obtain (40), we simply utilized the standard analysis of digital QAM systems in additive white Gaussian noise environments, e.g., see [87]. Since this theory is very well known, we omit the details of the derivation. Note that by including only intrinsic quantum noise in our quantum QAM link analysis, we are effectively analyzing a Gaussian channel since pure coherent states are Gaussian processes [2], [105].

Fig. 9 presents a comparison of error probability versus signal photons per symbol for classical and quantum QAM with 16-QAM modulation scenario. Results are qualitatively the same with higher values of $K$ and hence we omit these for brevity. It is clear from this figure that the quantum system outperforms the classical case since the quantum scheme can achieve the same probability of error of its classical counterpart under identical symbol rate but with smaller photon per symbol rate (hence less power is needed to support the same data rate with the quantum system than its classical counterpart.) The superiority of the quantum communication system in transmitting classical information over all-classical links has been also observed with other modulation types such as QPSK and PPM [106], [107].

\section{Applications to QuAntum Encryption}

Th quantum QAM communication system developed above can be expanded upon further to allow for security measures using Quantum Encryption. In particular we will examine how we can inject the BB84 algorithm into this system to transmit 


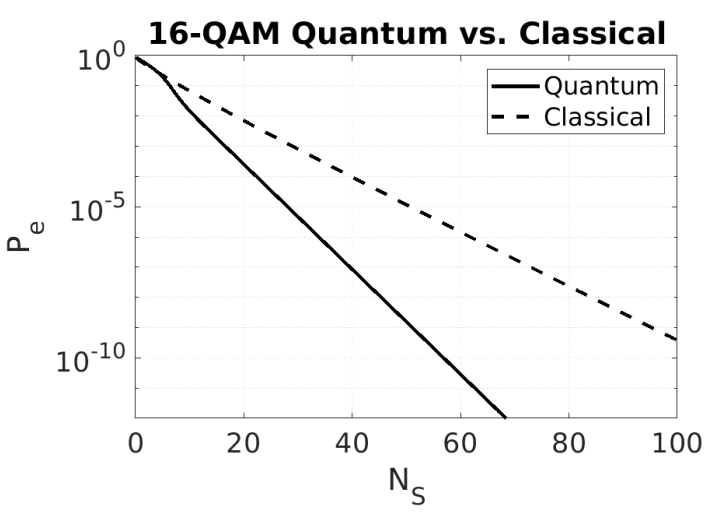

Fig. 9: Error probability $P_{e}$ vs. signal photons per symbol $N_{S}$ for classical and quantum 16-ary QAM.

encrypted information over the radiated q-antenna coherentstates.

\section{A. Overview of the BB84 Algorithm}

BB84 is a now classic quantum key distribution (QKD) protocol which takes advantage of Heisenberg's uncertainty principle and the no cloning theorem to provide a secure means for generating a symmetric encryption key [4], [6]. QKD protocols are information-theoretic key agreement schemes, where a random signal is generated by one party (Alice) and transmitted to another party (Bob) through a noisy channel. It works by choosing two orthogonal sets of quantum states to represent a binary stream of information. This can be done, for example, by using qubits in either the rectilinear or Hadamard basis. Denoting an arbitrary bit by $b \in\{0,1\}$, we establish two classical-to-quantum mappings: one for the Standard $(+)$ basis

$$
\begin{aligned}
& b=0 \mapsto\left|0^{+}\right\rangle=|0\rangle, \\
& b=1 \mapsto\left|1^{+}\right\rangle=|1\rangle,
\end{aligned}
$$

and one for the Hadamard $(x)$ basis

$$
\begin{aligned}
& b=0 \mapsto\left|0^{\times}\right\rangle=|+\rangle=\frac{1}{\sqrt{2}}(|0\rangle+|1\rangle), \\
& b=1 \mapsto\left|1^{\times}\right\rangle=|-\rangle=\frac{1}{\sqrt{2}}(|0\rangle-|1\rangle) .
\end{aligned}
$$

We begin with Alice, who will generate a random stream of $n$ bits $b_{i}, i \in\{1,2 \ldots n\}$, to send to Bob. She uses the quantum mappings (41) and (42) to encode the bits - a process called quantum encoding. We can write this process as

$$
b_{i} \mapsto\left|b_{i}^{j}\right\rangle,
$$

where the superscript $j \in\{+, \times\}$ denotes the basis which Alice encoded bit $i$ in. The mapping selected to encode each bit is randomly picked up by Alice, but also recorded by her. Once the quantum encoding is performed, Alice sends each quantum symbol one at a time to Bob. Bob needs two measurement apparatuses, denoted by observables $M^{+}$ and $M^{\times}$, to measure in either base. We can expand these observables into a sum of projectors scaled by eigenvalues

$$
M^{+}=\sum_{m} m P_{m}^{+}, M^{\times}=\sum_{m} m P_{m}^{\times},
$$

where $m=b+1$ and $P_{m}^{j}$ are projectors which form projector systems in their respective Hilbert Spaces. In other words,

$$
\prod_{m} P_{m}^{+}=\prod_{m} P_{m}^{\times}=0, \mathcal{H}_{j}=\sum_{m} P_{m}^{j}
$$

With this expansion, it follows that acting with $M^{j}$ on an incoming state $\left|b_{i}^{j}\right\rangle$, will produce an outcome of $m$. We may define a decision circuit, denoted by $D(\lambda)$, in order to simplify future computations based on the relation $b=D(m)=m-1$. Therefore, if the outcome is $m=2$ for example, the bit value would be 1 .

For optimal decision, it must be that

$$
P_{1}^{+}=|0\rangle\left\langle 0\left|, P_{2}^{+}=\right| 1\right\rangle\left\langle 1\left|, P_{1}^{\times}=\right|+\right\rangle\left\langle+\left|, P_{2}^{\times}=\right|-\right\rangle\langle-| \text {. }
$$

To show this, we consider the probability of measuring an outcome $m$ when applying one of these observables on an incoming state, namely

$$
p(m)=\left\langle b_{i}^{j}\left|P_{m}^{j}\right| b_{i}^{j}\right\rangle .
$$

When the state is in the + basis we have for the observable $M^{+}$

$$
\begin{aligned}
& p(m=1 \mid b=0)=\left\langle 0\left|P_{1}^{+}\right| 0\right\rangle=\langle 0 \mid 0\rangle\langle 0 \mid 0\rangle=1, \\
& p(m=2 \mid b=1)=\left\langle 1\left|P_{2}^{+}\right| 1\right\rangle=\langle 1 \mid 1\rangle\langle 1 \mid 1\rangle=1 .
\end{aligned}
$$

Since there's only two possible outcomes in each case, it follows that $p(1 \mid b=1)=p(2 \mid b=0)=0$. For the $\times$ basis, we have

$$
\begin{aligned}
& p(1 \mid b=0)=\left\langle+\left|P_{1}^{+}\right|+\right\rangle=1 / 2, \\
& p(2 \mid b=1)=\left\langle-\left|P_{2}^{+}\right|-\right\rangle=1 / 2 .
\end{aligned}
$$

This means that measuring with $M^{+}$in the $\times$basis will produce a random outcome. The same relations can be shown for the observable $M^{\times}$in the opposing bases.

From the above, if Alice and Bob both use the same basis - i.e., Alice encodes in the same base as what Bob measures in - then Bob will correctly decide what bit she sent with $100 \%$ certainty. On the other hand, if they choose opposing bases, Bob will decide the correct bit with $50 \%$ certainty based on the random outcome from his observable. Since Bob does not know what base Alice encodes each bit in, he is forced to randomly choose which apparatus to measure with at each instant. Once all qubits have been received, Bob communicates to Alice over a classical channel which basis he used for each qubit. Alice compares how each qubit was encoded to how they were measured, and discloses which bits Bob measured correctly. The qubits which were encoded by Alice in the same base which Bob measured in are used for the key, while the rest of the qubits are discarded. Security is maintained because, even if Eve hacked the classical channel all she would know is the bases which Bob measured in and which were measured correctly. Therefore she would only actually have the key if she happened to measure all the same bits correctly as what Bob did. Even then, she would have to not be detected by the bits used for comparison to detect her. 


\section{B. Eavesdropping on the Quantum Channel}

The fact that Alice and Bob discard the bits which were not encoded and measured in the same basis is important. This means that, in a noiseless channel, Alice and Bob should have the exact same bits, or a theoretical bit error rate (BER) of $100 \%$. They can use this as a means to detect someone listening on the quantum channel.

The BB84 protocol inherently provides means to detect eavesdroppers on the channel through quantum principles. Due to the no cloning theorem, an eavesdropper (Eve) cannot simply create her own replica of the qubit stream without disturbing the quantum states. Therefore, she must measure the qubits and then send a copy of whatever she measures to Bob. Since she has no knowledge of what basis the qubits are encoded in, she is forced to guess which basis to measure in just like Bob.

If Eve measures a qubit in a basis different from Alice, the bit will be randomized. She will choose the correct basis half the time, meaning half the bits will be the same but the other half will be randomized. This implies that $75 \%$ of the bits she sends to Bob will be the same as what Alice sent.

Alice and Bob can choose a subset of their bits in order to attempt to detect Eve. Since Eve will cause a bit to flip with a probability of $25 \%$, the more bits they use for detection the more likely they will be successful in detecting her. We can calculate the probability of detection as

$$
P_{D}=1-\left(\frac{3}{4}\right)^{n},
$$

where $n$ is the number of bits which Alice and Bob compare for this purpose. This equation basically says that for each bit they use for comparison, they have a $25 \%$ chance of detecting Eve if she's there.

\section{Quantum Cryptography Implementation}

The design of BB84 lends itself well to the use of a symmetric key encryption scheme. This is where Alice and Bob obtain a key which they both have and can use it to encrypt and decrypt their messages. The BB84 protocol can provide Alice and Bob with a shared key; recall that if they detect eavesdropping on the channel, they can immediately throw away the key and try again. With a sufficient number of bits used to detect the eavesdropper, they can know with high probability if somebody was listing in on the channel. For added security, they can repeat the process as many time as necessary to continuously produce new keys for communication. We only need to modify our system slightly to allow for an encrypted channel, shown in Fig. 10.

An example of a protocol which would work well with this design is the classical Data Encryption Standard (DES) encryption algorithm [9] in this simulation. DES is a 16-round Feistel Cipher which uses a 64-bit key; however, it has an effective key length of 56-bits because 8 are used as parity bits. Therefore, Alice and Bob can use BB84 to produce a 56bit key and then calculate the parity bits necessary for its use. Fig. 11 shows this curve analytically, and the results of a simulation for BB84 are plotted against to show the correlation.

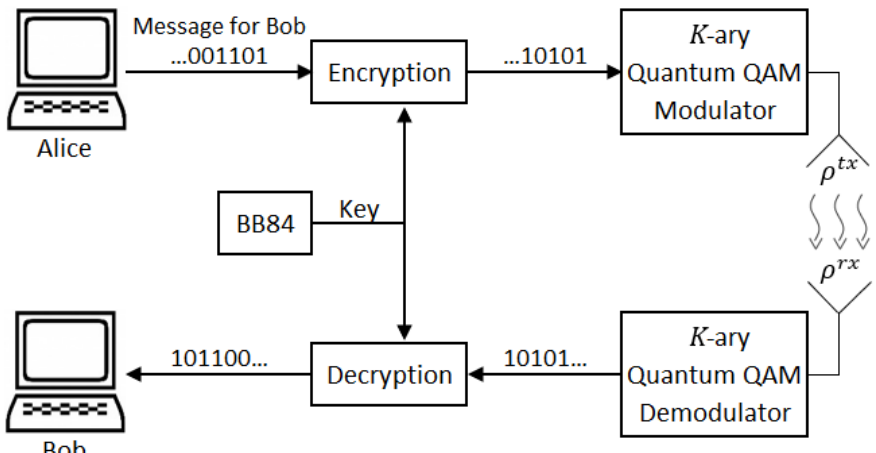

Fig. 10: Modified quantum communication system integrating BB84 in conjunction with a classical encryption method to provide security for the channel.

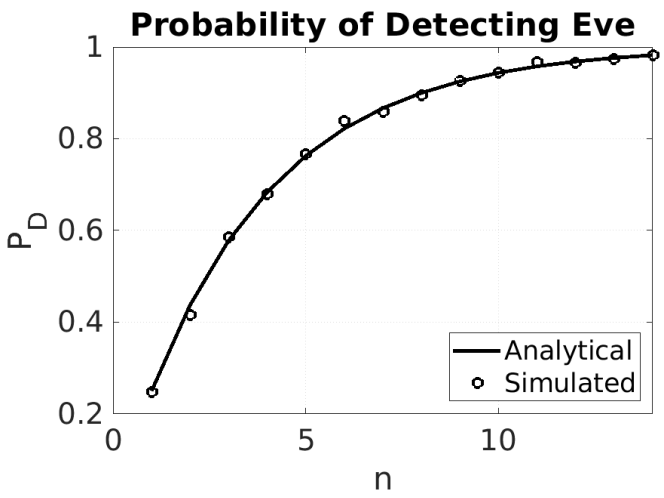

Fig. 11: The probability of detecting Eve depending on the number of bits. The simulation uses the quantum 64-QAM system discussed in the previous Sec. V after integration with the BB84 as per the blueprint in Fig. 10.

Excellent agreement is observed. The simulation involves the original quantum QAM system analyzed and implemented in Sec. IV above after integrating it with the BB84 encryption scheme. The probability of error was computed directly in the simulation by comparison with a randomly generated message at the front end and comparison with the exact analytical formula was made hence after. Only shot noise was included in this experiment.

\section{CONCLUSION}

We developed applications for coherent state representations of quantum radiation by optical antennas with focus on implementing secure $K$-ary digital communication links to improve spectral efficiency. The fundamental theory of coherent states and modulator and receiver design were developed from first principles (quantum mechanics and statistical decision theory). A concrete practical system utilizing a $K$-ary QAM quantum link was constructed and implemented through simulation of each stage. The overall performance of the system was monitored through suitable quantities such as probability of error and signal photon per symbol rates. It was found that increasing the spectral efficiency of the system (data rate per bandwidth) is possible through the use of high $K$-ary QAM with $K=64$. Moreover, comparison between the classical and 
the quantum version of the QAM system demonstrated consistent superiority of the quantum antenna link over links utilizing classical antennas. To ensure security, a standard quantum encryption algorithm, the BB84 method, was integrated with the proposed coherent-state quantum QAM system.

\section{REFERENCES}

[1] C. W. Helstrom, Quantum detection and estimation theory. Academic Press: Cambridge University Press, 1976.

[2] G. Cariolaro, Quantum communications. Springer, 2016.

[3] S. Imre and F. Azs, "Quantum computing and communications - an engineering approach," 012005.

[4] M. Nielsen and I. L. Chuang, Quantum computation and quantum information. Cambridge New York: Cambridge University Press, 2010.

[5] S. Barnett, "Optical demonstrations of statistical decision theory for quantum systems," Quantum Information Computation, vol. 4, pp. 450-459, 122004.

[6] S. M. Barnett, Quantum information. Oxford New York: Oxford University Press, 2009.

[7] G. Assche, Quantum cryptography and secret-key distillation. Cambridge: Cambridge University Press, 2006.

[8] D. Bernstein et al., Post-quantum cryptography. Berlin: Springer, 2009.

[9] J. Hoffstein, J. Pipher, and J. H. Silverman, An introduction to mathematical cryptography. New York: Springer, 2014.

[10] E. Rieffel and W. Polak, Quantum Computing: A Gentle Introduction. MIT Press, 2014.

[11] M. Wilde, Quantum information theory. Cambridge, UK New York: Cambridge University Press, 2017.

[12] S. A. Schelkunoff and H. T. Friss, Antennas: Theory and practice. New York; Chapman \& Hall: London, 1952.

[13] C. A. Balanis, Antenna Theory: Analysis and Design, 4th ed. Interscience: Wiley, 2015.

[14] W. C. Chew, Waves and Fields in Inhomogenous Media. Wiley-IEEE, 1999.

[15] L. Felsen, Radiation and Scattering of Waves. Piscataway, NJ: IEEE Press, 1994.

[16] S. Mikki and Y. Antar, New Foundations for Applied Electromagnetics: The Spatial Structure of Fields. London: Artech House, 2016.

[17] — - "On the Fundamental Relationship Between the Transmitting and Receiving Modes of General Antenna Systems: A New Approach," IEEE Antennas and Wireless Propagation Letters, vol. 11, pp. 232-235, 2012.

[18] C. Mead, Collective electrodynamics: quantum foundations of electromagnetism. Cambridge, Mass: MIT Press, 2000.

[19] D. Grimes and C. A. Grimes, The electromagnetic origin of quantum theory and light. Hackensack, N.J: World Scientific, 2005.

[20] W. C. Chew, A. Y. Liu, C. Salazar-Lazaro, and W. E. I. Sha, "Quantum electromagnetics: A new look-part I," IEEE Journal on Multiscale and Multiphysics Computational Techniques, vol. 1, pp. 73-84, 2016.

[21] _ -, "Quantum electromagnetics: A new look—part II," IEEE Journal on Multiscale and Multiphysics Computational Techniques, vol. 1, pp. 85-97, 2016

[22] W. E. I. Sha, A. Y. Liu, and W. C. Chew, "Dissipative quantum electromagnetics," IEEE Journal on Multiscale and Multiphysics Computational Techniques, vol. 3, pp. 198-213, 2018.

[23] W. C. Chew, W. E. I. Sha, and Q. I. Dai, "Green's dyadic, spectral function, local density of states, and fluctuation dissipation theorem," Progress In Electromagnetics Research, vol. 166, pp. 147-165, 2019.

[24] T. E. Roth and W. C. Chew, "Role of classical time domain CEM methods for quantum electromagnetics," in 2019 IEEE International Symposium on Antennas and Propagation and USNC-URSI Radio Science Meeting, 2019, pp. 1063-1064.

[25] D.-Y. Na, J. Zhu, W. C. Chew, and F. L. Teixeira, "Quantum information preserving computational electromagnetics," Phys. Rev. A, vol. 102, p. 013711, Jul 2020.

[26] D.-Y. Na and W. C. Chew, "Classical and quantum electromagnetic interferences: What is the difference?" Progress In Electromagnetics Research, vol. 168, pp. 1-13, 2020.

[27] G. W. Hanson, "Aspects of quantum electrodynamics compared to the classical case: Similarity and disparity of quantum and classical electromagnetics," IEEE Antennas and Propagation Magazine, vol. 62, no. 4, pp. 16-26, 2020.
[28] S. Mikki, "Exact derivation of the radiation law of antennas embedded into generic nonlocal metamaterials: A momentum-space approach," in 2020 14th European Conference on Antennas and Propagation (EuCAP), 2020, pp. 1-5.

[29] S. Mikki, "Theory of electromagnetic radiation in nonlocal metamaterials - Part I: Foundations," Progress In Electromagnetics Research B, vol. 89, pp. 63-86, 2020 .

[30] _ _ "Theory of electromagnetic radiation in nonlocal metamaterials - Part II: Applications," Progress In Electromagnetics Research B, vol. 89, pp. 87-109, 2020.

[31] S. Mikki and A. Kishk, "Electromagnetic wave propagation in nonlocal media: Negative group velocity and beyond," Progress In Electromagnetics Research B, vol. 14, pp. 149-174, 2009.

[32] _ - "Nonlocal electromagnetic media: A paradigm for material engineering," in Passive Microwave Components and Antennas. InTech, April 2010.

[33] S. Mikki and Y. Antar, "Critique of antenna fundamental limitations," in 2010 URSI International Symposium on Electromagnetic Theory, Aug 2010, pp. 122-125.

[34] S. Mikki and Y. Antar, "A theory of antenna electromagnetic near field-part I," IEEE Transactions on Antennas and Propagation, vol. 59, no. 12, pp. 4691-4705, December 2011.

[35] S. M. Mikki and Y. M. M. Antar, "A theory of antenna electromagnetic near field - part II," IEEE Transactions on Antennas and Propagation, vol. 59, no. 12, pp. 4706-4724, Dec 2011

[36] S. Mikki and Y. Antar, "A rigorous approach to mutual coupling in general antenna systems through perturbation theory," IEEE Antennas and Wireless Communication Letters, vol. 14, pp. 115-118, 2015.

[37] S. M. Mikki and Y. M. M. Antar, "A new technique for the analysis of energy coupling and exchange in general antenna systems," IEEE Transactions on Antennas and Propagation, vol. 63, no. 12, pp. 55365547, Dec 2015.

[38] S. Clauzier, S. Mikki, and Y. Antar, "Design of near-field synthesis arrays through global optimization," IEEE Transactions on Antennas and Propagation, vol. 63, no. 1, pp. 151-165, Jan 2015.

[39] S. Mikki, D. Sarkar, and Y. Antar, "Near-field cross-correlation analysis for MIMO wireless communications," IEEE Antennas and Wireless Propagation Letters, vol. 18, no. 7, pp. 1357-1361, July 2019.

[40] S. Mikki, S. Clauzier, and Y. Antar, "A correlation theory of antenna directivity with applications to superdirective arrays," IEEE Antennas and Wireless Propagation Letters, vol. 18, no. 5, pp. 811-815, May 2019.

[41] D. Sarkar, S. Mikki, K. V. Srivastava, and Y. Antar, "Dynamics of antenna reactive energy using time-domain IDM method," IEEE Transactions on Antennas and Propagation, vol. 67, no. 2, pp. 1084 1093, Feb 2019.

[42] D. Sarkar, S. Mikki, A. Alzahed, K. V. Srivastava, and Y. Antar, "New considerations on electromagnetic energy in antenna near-field by timedomain approach," in 2017 IEEE Applied Electromagnetics Conference (AEMC), Dec 2017, pp. 1-2.

[43] S. Mikki, D. Sarkar, and Y. Antar, "Beyond antenna Q: On reactive energy and the need for a spatio-temporal dynamical paradigm," in 2019 13th European Conference on Antennas and Propagation (EuCAP), March 2019, pp. 1-5.

[44] S. Mikki, D. Sarkar, and Y. Antar, "On localized antenna energy in electromagnetic radiation," Progress In Electromagnetics Research M, vol. 79, pp. 1-10, 2019

[45] S. Mikki and Y. Antar, "Aspects of generalized electromagnetic energy exchange in antenna systems: A new approach to mutual coupling," EuCap 2015, April 2015.

[46] O. Keller, Quantum Theory of Near-Field Electrodynamics. Berlin New York: Springer, 2011.

[47] S. Mikki, "Theory of nonsinusoidal antennas for near-field communication system analysis," Progress In Electromagnetics Research, vol. 86, pp. 177-193, 2020.

[48] D. Sarkar, S. M. Mikki, and Y. M. M. Antar, "Poynting localized energy: Method and applications to gain enhancement in coupled antenna systems," IEEE Transactions on Antennas and Propagation, vol. 68 , no. 5, pp. 3978-3988, 2020

[49] L. Novotny, Principles of Nano-Optics. Cambridge: Cambridge University Press, 2012.

[50] K. Cho, Optical response of nanostructures: microscopic nonlocal theory. Berlin New York: Springer, 2003.

[51] S. Mikki and A. Kishk, "Effective medium theory for carbon nanotube composites and their potential applications as metamaterials," in 2007 IEEE/MTT-S International Microwave Symposium, June 2007, pp. $1137-1140$. 
[52] S. Mikki and A. Kishk, "Theory of optical scattering by carbon nanotubes," Microwave and Optical Technology Letters, vol. 49, no. 10, pp. 2360-2364, Jul. 2007.

[53] S. Mikki and A. Kishk, "Mean-field electrodynamic theory of aligned carbon nanotube composites," IEEE Transactions on Antennas and Propagation, vol. 57, no. 5, pp. 1412-1419, May 2009.

[54] T. Anderson, Plasma antennas. Boston: Artech House, 2011.

[55] J. N. Farahani, D. W. Pohl, H.-J. Eisler, and B. Hecht, "Single quantum dot coupled to a scanning optical antenna: A tunable superemitter," Phys. Rev. Lett., vol. 95, p. 017402, Jun 2005.

[56] R. Filter, S. Mühlig, T. Eichelkraut, C. Rockstuhl, and F. Lederer, "Controlling the dynamics of quantum mechanical systems sustaining dipole-forbidden transitions via optical nanoantennas," Phys. Rev. B, vol. 86, p. 035404, Jul 2012.

[57] G. Y. Slepyan and A. Boag, "Quantum nonreciprocity of nanoscale antenna arrays in timed dicke states," Phys. Rev. Lett., vol. 111, p. 023602, Jul 2013.

[58] P. E. Kremer, A. C. Dada, P. Kumar, Y. Ma, S. Kumar, E. Clarke, and B. D. Gerardot, "Strain-tunable quantum dot embedded in a nanowire antenna," Phys. Rev. B, vol. 90, p. 201408, Nov 2014.

[59] J. Liu, M. Zhou, L. Ying, X. Chen, and Z. Yu, "Enhancing the optical cross section of quantum antenna," Phys. Rev. A, vol. 95, p. 013814 Jan 2017

[60] J. M. Fitzgerald, S. Azadi, and V. Giannini, "Quantum plasmonic nanoantennas," Phys. Rev. B, vol. 95, p. 235414, Jun 2017.

[61] S. Mikki, "Quantum antenna theory," in 2017 IEEE AP-S Symposium on Antennas and Propagation and USNC-URSI Radio Science Meeting July 9-14, 2017 San Diego, California, USA. IEEE Antennas \& Propagation Society, 2017.

[62] C. Müller, J. Combes, A. R. Hamann, A. Fedorov, and T. M. Stace, "Nonreciprocal atomic scattering: A saturable, quantum Yagi-Uda antenna," Phys. Rev. A, vol. 96, p. 053817, Nov 2017.

[63] A. Komarov and G. Slepyan, "Quantum antenna as an open system Strong antenna coupling with photonic reservoir," Applied Sciences, vol. 8, no. 6, p. 951, Jun. 2018

[64] A. Mikhalychev, D. Mogilevtsev, G. Y. Slepyan, I. Karuseichyk, G. Buchs, D. L. Boiko, and A. Boag, "Synthesis of quantum antennas for shaping field correlations," Phys. Rev. Applied, vol. 9, p. 024021, Feb 2018.

[65] I. n. Liberal, I. n. Ederra, and R. W. Ziolkowski, "Quantum antenna arrays: The role of quantum interference on direction-dependent photon statistics," Phys. Rev. A, vol. 97, p. 053847, May 2018.

[66] _ - "Control of a quantum emitter's bandwidth by managing its reactive power," Phys. Rev. A, vol. 100, p. 023830, Aug 2019.

[67] S. Mikki, "Quantum antenna theory for secure wireless communications," in 2020 14th European Conference on Antennas and Propagation (EuCAP), 2020, pp. 1-4.

[68] S. Mikki, "A quantum MIMO architecture for antenna wireless digital communications," Progress In Electromagnetics Research C, vol. 93 pp. 143-156, 2019.

[69] M. Agio et al., Optical Antennas. Cambridge: Cambridge University Press, 2012.

[70] R. Glauber, Quantum theory of optical coherence: selected papers and lectures. Weinheim: Wiley-VCH, 2007.

[71] J. Klauder and E. C. G. Sudarshan, Fundamentals of quantum optics. Mineola, N.Y: Dover Publications, 2006.

[72] E. Schrodinger, Collected papers on wave mechanics. American Mathematical Society, 2003.

[73] E. Sudarshan, "Equivalence of semiclassical and quantum mechanical descriptions of statistical light beams," Physical Review Letters, vol. 10, pp. 277-279, 1963.

[74] C. L. Mehta and E. C. G. Sudarshan, "Relation between quantum and semiclassical description of optical coherence," Phys. Rev., vol. 138, pp. B274-B280, Apr 1965.

[75] C. Mehta and E. Sudarshan, "Time evolution of coherent states," Physics Letters, vol. 22, no. 5, pp. 574 - 576, 1966.

[76] C. L. Mehta, P. Chand, E. C. G. Sudarshan, and R. Vedam, "Dynamics of coherent states," Phys. Rev., vol. 157, pp. 1198-1206, May 1967.

[77] J. Klauder, Coherent states: applications in physics and mathematical physics. Singapore: World Scientific, 1985.

[78] J. C. Garrison and R. Chiao, Quantum optics. Oxford: Oxford University Press, 2014.

[79] L. Mandel and E. Wolf, Optical coherence and quantum optics. Cambridge: Cambridge University Press, 1995.

[80] A. Dasgupta, M.-M. Mennemanteuil, M. Buret, N. Cazier, G. C. des Francs, and A. Bouhelier, "Optical wireless link between a nanoscale antenna and a transducing rectenna," Nature Communications, vol. 9 , no. 1, May 2018.

[81] R. F. Oulton, V. J. Sorger, T. Zentgraf, R.-M. Ma, C. Gladden, L. Dai, G. Bartal, and X. Zhang, "Plasmon lasers at deep subwavelength scale," Nature, vol. 461, no. 7264, pp. 629-632, Aug. 2009.

[82] A. A. Arisheh, S. Mikki, and N. Dib, "Design of transmitting nanodipole antenna using a subwavelength laser excitation method," in 2019 IEEE International Symposium on Antennas and Propagation and USNC-URSI Radio Science Meeting, 2019, pp. 1313-1314.

[83] — - "A subwavelength-laser-driven transmitting optical nanoantenna for wireless communications," IEEE Journal on Multiscale and Multiphysics Computational Techniques, vol. 5, pp. 144-154, 2020.

[84] B. Saleh and M. Teich, Fundamentals of photonics. Hoboken, N.J: Wiley-Interscience, 2007.

[85] E. Merzbacher, Quantum mechanics. New York: Wiley, 1998.

[86] S. Haroche and J.-M. Raimond, Exploring the quantum: atoms, cavities and photons. Oxford New York: Oxford University Press, 2006.

[87] B. P. Lathi and Z. Ding, Modern digital and analog communication systems. New York: Oxford University Press, 2019.

[88] R. Heath, Foundations of MIMO communication. Cambridge, United Kingdom New York, NY, USA: Cambridge University Press, 2019.

[89] S. Mikki, A. Hanoon, J. Aulin, and Y. Antar, "The time-dependent ACGF with applications to M-ary digital communication systems," in The 11th European Conference on Antennas and Propagation (EuCap 2017), 2017, pp. 19-24.

[90] J. Neumann, Mathematical foundations of quantum mechanics. Princeton: Princeton University Press, 2018.

[91] P. A. M. Dirac, The principles of quantum mechanics. Oxford England: Clarendon Press, 1981.

[92] D. Bohm, Quantum theory. New York: Dover Publications, 1989.

[93] H. Yuen, R. Kennedy, and M. Lax, "Optimum testing of multiple hypotheses in quantum detection theory," IEEE Transactions on Information Theory, vol. 21, no. 2, pp. 125-134, 1975.

[94] E. Zeidler, Quantum field theory I: Basics in Mathematics and Physics. Springer, 2009

[95] S. Hassani, Mathematical physics: a modern introduction to its foundations. Cham: Springer, 2013.

[96] C. W. Helstrom, J. W. S. Liu, and J. P. Gordon, "Quantum-mechanical communication theory," Proceedings of the IEEE, vol. 58, no. 10, pp. $1578-1598,1970$

[97] A. Holevo, "Statistical decision theory for quantum systems," Journal of Multivariate Analysis, vol. 3, pp. 337-394, 121973.

[98] R. Kennedy, "A near-optimum receiver for the binary coherent state quantum channel," M.I.T. Res. Lab. Electron. Qt. Prog. Rep., vol. 108 , pp. 219-225, 011973.

[99] A. Assalini, G. Cariolaro, and G. Pierobon, "Efficient optimal minimum error discrimination of symmetric quantum states," Physical Review A, vol. 81, Jan 2010

[100] P. Hausladen, R. Jozsa, B. Schumacher, M. Westmoreland, and W. K. Wootters, "Classical information capacity of a quantum channel," Phys. Rev. A, vol. 54, pp. 1869-1876, Sep 1996.

[101] Y. C. Eldar and G. D. Forney, "On quantum detection and the squareroot measurement," IEEE Transactions on Information Theory, vol. 47, no. 3 , pp. $858-872,2001$

[102] G. Cariolaro, R. Corvaja, and G. Pierobon, "Compression of pure and mixed states in quantum detection," Proceedings of the Global Communications Conference, GLOBECOM, pp. 1-5, December 2011.

[103] A. Hanoon and S. Mikki, "Bandwidth-enhancement of digital communication systems employing narrowband antennas: A novel electromagnetic OFDM approach," in 2017 IEEE International Symposium on Antennas and Propagation USNC/URSI National Radio Science Meeting, July 2017, pp. 527-528.

[104] S. Mikki, A. Hanoon, J. Persano, A. Alzahed, Y. Antar, and J. Aulin, "Theory of electromagnetic intelligent agents with applications to MIMO and DoA systems," in 2017 IEEE International Symposium on Antennas and Propagation USNC/URSI National Radio Science Meeting, July 2017, pp. 525-526.

[105] A. Holevo and V. Giovannetti, "Quantum channels and their entropic characteristics," Reports on progress in physics. Physical Society (Great Britain), vol. 75, p. 046001, April 2012.

[106] G. Cariolaro and G. Pierobon, "Theory of quantum pulse position modulation and related numerical problems," IEEE Transactions on Communications, vol. 58, no. 4, pp. 1213-1222, 2010.

[107] — - "Performance of quantum data transmission systems in the presence of thermal noise," IEEE Transactions on Communications, vol. 58, no. 2, pp. 623-630, 2010. 